\section{Omega-6/omega-3 essential fatty acid ratio: the scientific evidence}

A. P. Simopoulos and L. G. Cleland, eds. Karger; World Review of Nutrition and Dietetics 2003, Vol. 92.174 pp. ISBN 3-8055-7640-4. EUR 163.-IUSD 198.-

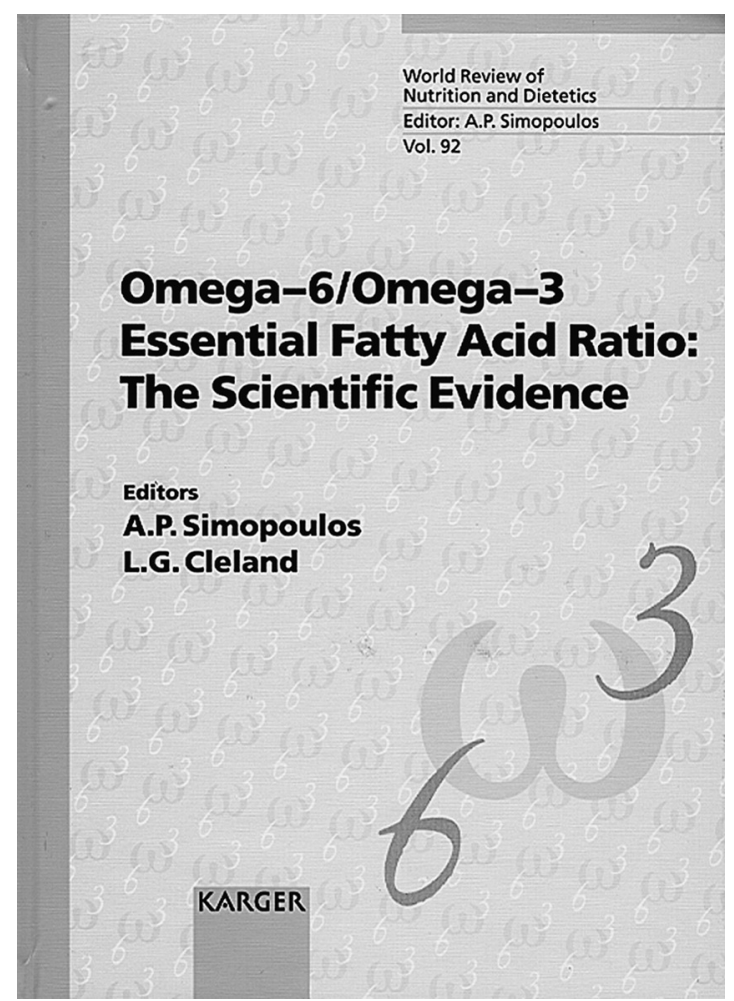

Both the amount and the ratio of n- 6 and n-3 polyunsaturated fatty acids in the diet play independent roles in health and disease. This is well documented by the editors and coauthors in the 10 chapters of the book presenting the importance of the $n-6 / n-3$ fatty acid ratio for preventing diseases such as coronary heart disease (CHD), cancer and arthritis, and for proper brain development at an early age, ageing and degenerative diseases of the brain. A presentation is also given of whether the n-3 fatty acid $\alpha$-linolenic acid has an independent role as an essential fatty acid, or only as a precursor of eicosanoids. In this book the "omega" nomenclature of essential fatty acids, as coined by the pioneer Ralph Holman, is used, even though the " $n$ " nomenclature is most commonly used today.

Simopoulos presents the evolutionary aspect of the ratio of dietary $n-6$ and $n-3$ fatty acids from the time of our ancestors during the Palaeolithic period with a n-6/n-3 fatty acid ratio of about $1 / 1$, to modern times, with a dietary ratio in developed and developing countries at a level between 5/1 and 50/1. Some of the most common chronic diseases of today are CHD, diabetes, obesity, autoimmune diseases, arthritis and depression, and they are all associated with an increased production of thromboxane $A_{2}$, leukotriene $B_{4}$ and some cytokines. These factors increase with increased dietary intake of n-6 fatty acids, whereas they decrease with increased intake of $n-3$ fatty acids. Thus, a low dietary n-6/n-3 fatty acid ratio is beneficial for preventing these conditions, but the optimal ratio varies from $1 / 1$ to $4 / 1$ depending on which disease is studied. In contrast, a high ratio of dietary n-6/n-3 fatty acids is strongly associated with an increased risk of many chronic diseases. The appendix to this chapter presents the recommended dietary intakes of n-6 and n-3 fatty acids for adults and infants as stated by an international meeting of scientists at the National Institutes of Health in Bethesda, USA. An adequate intake of linoleic acid, $4.44 \mathrm{~g} \mathrm{day}^{-1}$, and $\alpha$-linolenic acid, $2.22 \mathrm{~g}$ day $^{-1}$, giving a ratio $2 / 1$, is recommended by this group. An intake of $0.65 \mathrm{~g} \mathrm{day}^{-1}$ of eicosapentaenoic acid (EPA) plus docosahexaenoic acid (DHA) (very long-chain polyunsaturated n-3 fatty acids) is also recommended.

Cleland et al. present the beneficial effect of n-3 fatty acids and fish oil on arthritis and related diseases. The replacement of n-6 fatty acids with n-3 and monounsaturated fatty acids which are not precursors of proinflammatory eicosanoids, reduces the risk for development of arthritis, and also provides relief when it occurs. A daily dose of $3 \mathrm{~g}$ $\mathrm{EPA}+\mathrm{DHA}$ is recommended for anti-inflammatory effects.

An interesting approach to obtain a balanced n-6/ n-3 fatty acid ratio in mammalian tissues was obtained by Kang, who gives a presentation of gene transfer of the enzyme missing in mammalian organisms, n-3 desaturase (delta-15 desaturase) from a roundworm to cultured mammalian cells. The enzyme introduces a double bond in the n-3 position of linoleic acid to give $\alpha$-linolenic acid until a ratio of $1 / 1$ is obtained.

Hamazaki and Okuyama give a thorough review of international studies on the role of n- 6 and n-3 
fatty acids in the prevention of chronic diseases, for the Japan Society for Lipid Nutrition. During the past few decades increasing amounts of linoleic acid intake along with decreasing saturated fatty acids, have been the major basis in lipid nutrition for prevention of CHD in Japan. The authors propose that a linoleic acid intake of $3-4 E \%$ is adequate for people ingesting $\mathrm{n}-3$ fatty acids at the average Japanese level $\left(0.6 E^{\%} \% \alpha\right.$-linolenic acid and $0.5 E \%$ $\mathrm{EPA}+\mathrm{DHA})$.

The book is recommended, as it gives the latest information on dietary n- 6 and n-3 fatty acids and their relationship to health and disease. It should be mentioned that the first section of the preface states that the $n-6 / n-3$ fatty acid ratio of Western diets is 15/1-16.7/1. This may be true for the USA, but for most European countries the ratio is lower, and in Nordic countries it is below 5/1 (Eur J Clin Nutr 1999;53:143-57).

Erling N. Christiansen

Institute for Nutrition Research, University of Oslo, Norway

E-mail: e.n.christiansen@basalmed.uio.no 